\title{
La dialisii che sceglierei nel 2001
}

\author{
Angela Rosa Pinciaroli
}

\author{
Divisione di Nefrologia e Dialisi, Azienda Ospedaliera "Pugliese Ciaccio", Catanzaro
}

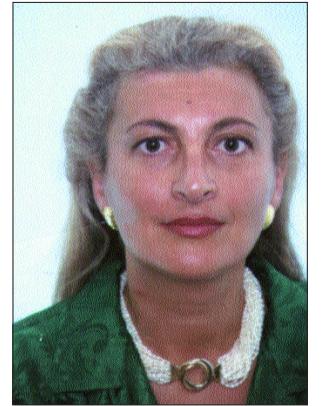

i v e r e,
giorno dopo
giorno, accanto
a persone in
trattamento dia-
litico fa riflette-
re su come sa-
rebbe la "tua"
vita se ti trovas-
si a dover (e mi auguro di continuare a dire "poter") scegliere una metodica dialitica che ti consente di vivere ma che, comunque, cambia il tuo stile di vita e fa nascere il timore della perdita del proprio ruolo nella società, nella famiglia, con il proprio partner e correre il rischio di un diverso rapporto con il mondo del lavoro e di insicurezza economica.

La risposta a un dramma umano così forte deve essere data dal nefrologo con una terapia che consenta di vivere quanto più a lungo possibile (1), ma anche nel miglior modo possibile con certezze e speranze nel futuro.

Non basta garantire una dialisi comunque e qualunque! La validità di ogni intervento terapeutico, dialisi inclusa, si misura non solo in termini di benessere fisico recuperato dal soggetto trattato, ma anche di benessere psicosociale e di conservata capacità al lavoro fisico e mentale.

Quanto maggiore sarà il recupero, tanto minore sarà il "costo" che la società dovrà sostenere per quell'individuo.

Esiste un'espansione della spesa sanitaria, specie nel settore della dialisi, con prevedibili incrementi nel futuro per l'aumento del numero delle persone che necessitano di trattamento dialitico sia per l'aumento della vita media della popolazione sia per gli ottimi risultati ottenuti nel trattamento di pazienti affetti da diabete, patologie cardiovascolari o altre patologie severe, grazie anche all'impiego di metodiche dialitiche che si avvalgono di tecnologie sempre più avanzate, ma anche più costose.

Bisogna tuttavia sempre ricordare che al centro delle valutazioni che portano alla scelta di una metodica dialitica deve sempre essere posta la "persona".

Oltre al diritto di ognuno di noi a essere curato (e spero si possa decidere anche da chi, nel rispetto della volontà individuale), ci sono principi etici che devono guidare il nostro operato e che non possono essere modificati da motivi puramente economici.

Non si può correre il rischio che l'aspetto economico venga considerato elemento dominante di alcune scelte che riguardano la metodica dialitica e/o possibili criteri di selezione nell'ammissione al trattamento dialitico stesso.

Ogni Centro perciò ha il dovere (e diritto) di proporre tutte le metodiche dialitiche disponibili per fare poi la scelta più adatta a risolvere $\mathrm{i}$ problemi clinici e ottenere la massima riabilitazione possibile nei singoli pazienti (2), qualità nel vivere e possibile reinserimento nel ciclo produttivo della società.

Convinti che la dialisi domiciliare possa, più di ogni altra modalità di trattamento dell'uremia terminale, raggiungere questi obiettivi, abbiamo avviato nel 1985, in un contesto culturale e sociale, in cui la proposta di emodialisi domiciliare era stata fino ad allora impensabile, un programma di emodialisi domiciliare, attratti da uno schema emodialitico giornaliero breve (due ore), che già Buoncristiani, a Perugia, portava avanti con successo. La nostra speranza era che la proposta di un trattamento emodialitico breve, anche se giornaliero, da poter effettuare a casa propria, nelle ore della giornata libere da impegni per il dializzato e per il suo partner, potesse suscitare interesse. Così è stato. In realtà con tale metodica $\mathrm{i}$ pazienti so- 
no"sicuri" di poter affrontare il trattamento domiciliare dopo aver constatato, durante il periodo di addestramento in Centro, la pressoché totale assenza di sintomi intradialitici (a esempio, ipotensioni) e ciò per la brevità del trattamento, ma soprattutto per l'incremento ponderale interdialitico meglio controllato, le oscillazioni elettrolitiche e osmotiche più contenute, un equilibrio acido-base ben controllato, una rimozione giornaliera di tossine. La possibilità di dializzare nella propria famiglia, in un normale svolgersi delle giornate che contemplano l'impegno di dializzare, in un periodo scelto e in un'unica soluzione temporale di circa due ore, con un partner che garantisce la sua presenza "in vicinanza" ma che, spesso, può svolgere altri compiti contemporaneamente, si addice a persone di tutte le età, giovani e anziani, uomini e donne di qualsiasi estrazione sociale ed economica. Dall'architetto, sindaco della nostra città, che riusciva a conciliare il suo duplice impegno lavorativo, alla signora quasi ottantenne con la ritrovata voglia di occuparsi della casa e il piacere del nuoto; dal giovane meccanico che porta avanti un pesante lavoro di officina al bancario che normalmente svolge il suo lavoro, alla giovane donna che, oltre a occuparsi della famiglia e aiutare il marito agricoltore nel lavoro dei campi, corona il desiderio di maternità e, dopo la perdita di una bimba nata prematura, adotta la figlia desiderata (3).

Nessun sostegno assistenziale per i pazienti in dialisi o economico per i loro partner.

Molte le gratificazioni umane e professionali per noi medici e infermieri: siamo riusciti ad avviare un nuovo programma dialitico in cui crediamo, capace di cambiare nei nostri pazienti, nelle loro famiglie, nel loro ambiente di lavoro, l'idea del dializzato persona "comunque malata" e soggetto passivo con l'obiettivo della "pensione" come destino definitivo; finalmente la persona dializzata diventa soggetto attivo e produttivo, svolgendo il proprio lavoro nella società e in grado di compensare il costo della sua malattia.
Il trattamento emodialitico giornaliero domiciliare, anche protratto per molti anni, non ha mai portato in alcuno dei nostri pazienti il desiderio di passare o ritornare al trattamento trisettimanale.

In realtà l'emodialisi giornaliera determina una maggiore condizione di "fisiologicità" nel nostro organismo, garantendo un "milieu intérieur" più stabile e meno intriso nella condizione uremica, che rende ragione dei suoi molti effetti positivi. Lo schema giornaliero è di fondamentale importanza se si considera che tutti i progressi tecnologici raggiunti nelle metodiche dialitiche, in questi anni, non sono riusciti a modificare sostanzialmente l'aspetto di "non fisiologicità" del trattamento emodialitico trisettimanale.

Possiamo dire, dopo sedici anni di esperienza, che numerosi sono i risultati positivi ottenuti con l'emodialisi giornaliera: ottimo controllo della pressione arteriosa, miglioramento dell'anemia, riduzione dell'ipertrofia cardiaca, miglioramento del profilo ormonale, della libido, della funzione sessuale, ottimo stato nutrizionale, una dieta pressoché libera, un miglior stile di vita, un netto miglioramento delle funzioni fisiche, intellettuali e della qualità della vita, costi più bassi $(3,4)$.

I costi più contenuti riconosciuti alla emodialisi domiciliare $(5,6)$ sono favoriti nella emodialisi giornaliera, oltre che dalla sua minore interferenza con la vita lavorativa del soggetto dializzato e del suo partner, dall'assenza di un "tempo di recupero" in post-dialisi e, soprattutto, da un minor impiego di farmaci (antiipertensivi, Eritropoietina ecc.) e da minore ospedalizzazione (7). Minori costi dunque nel trattamento dialitico domiciliare associato a una notevole qualità di vita, un rapporto costo/beneficio decisamente favorevole in questa terapia.

Di fronte a una lievitazione della spesa nel settore dialitico, dato che è eticamente improponibile negare l'accesso in dialisi poiché un modo di vivere non soddisfacente per un individuo può esserlo invece per un altro, $\mathrm{e}$ di fronte a una disponibilità di risorse economiche immodificata, se non in fase di restrizione, l'unica via percorribile è quella della "corretta gestione delle risorse umane (personale medico e infermieristico, Pazienti-Utenti), strutturali ed economiche", disponibili (8).

Al momento, a mio avviso, l'opportunità più concreta viene offerta ai nefrologi dalla dialisi domiciliare, utilizzata anche attraverso programmi di dialisi domiciliare integrata, nonostante la difficoltà di operare in alcuni contesti culturali, sociali, politici e amministrativi.

Bisognerà diffondere la dialisi domiciliare con ogni sforzo. Per fare ciò è necessario che a livello politico e amministrativo vi sia la sensibilità, il reale interesse e la lungimiranza di intervenire per regolare, con criteri di equità, la distribuzione delle risorse economiche disponibili. Ogni Centro di nefrologia e di dialisi deve essere messo in condizione di poter avviare un programma di emodialisi domiciliare attraverso l'assegnazione al proprio budget di somme di entità proporzionale al numero dei soggetti che avvia al trattamento domiciliare, alla tipologia dei pazienti (giovani, anziani, contesto familiare, con o senza disponibilità di partner), alla qualità della prestazione fornita, al grado di riabilitazione raggiunto, alla riduzione della spesa farmacologica e della ospedalizzazione ecc. Con meccanismo "premiante" non solo la quantità ma anche, e soprattutto, la qualità del risultato raggiunto. Al nefrologo si chiede, prima di tutto, la perdita di ogni pregiudizio verso l'emodialisi domiciliare e la capacità di individuare i soggetti che, oltre che per le condizioni cliniche, per la loro personalità, abitudini di vita, necessità di indipendenza e sicurezza decisionale e/o altre motivazioni, siano da avviare al trattamento domiciliare. Dovrà sapere proporre in maniera convinta la metodica dialitica, infondendo sicurezza nella pratica domiciliare, coadiuvato in questo da personale infermieristico opportunamente addestrato e impiegato anche nel controllo domiciliare dei pazienti trattati o come supporto al partner.

La creazione di progetti obiettivi che 
prevedano anche gratificazioni economiche per il personale potrebbe favorire l'impegno nella diffusione dell'emodialisi domiciliare. Opportuno l'impiego di volontari addestrati.

Al paziente-utente si chiede di essere soggetto attivo della sua vita, nella sua famiglia, nella società ma dovrebbe poter contare su incentivi per il partner e avere così una tranquillità nella continuità della scelta effettuata. Il risparmio, derivante dalla minore spesa consentita dalla emodialisi domiciliare, dovrebbe essere assegnato al Centro che ha avviato e segue il trattamento domiciliare. Tale risparmio dovrebbe consentire a ogni Centro, attraverso reinvestimenti - autofinanziamenti, di fare fronte alle crescenti richieste di dialisi, consentire il trattamento di quei pazienti che per problemi organizzativi e/o familiari insormontabili non possono essere deospedalizzati e di quei pazienti che, per le loro condizioni cliniche, possono essere dializzati soltanto in Centro e, se severamente impegnati, richiedono grande sforzo organizzativo, impiego di personale e supporto farmacologico.

In particolare i Centri chiamati a sostenere l'onere di trattare i pazienti affetti da patologie più gravi devono essere messi in condizione di operare anche attraverso un programma di dialisi domiciliare per giungere, con una corretta gestione, almeno a un parziale autofinanziamento.

Sicuramente mi sento di consigliare, per quanti affetti da insufficienza renale terminale che siano in condizioni di poterlo fare, un trattamento dialitico domiciliare, perché sarebbe quello che io sceglierei, se mai fossi nella condizione di dover scegliere.

Considerata la nostra esperienza positiva, e con la disponibilità di un partner, sceglierei l'emodialisi giornaliera perché ritengo tale metodica più adattabile alle esigenze di vita, oltre agli indubbi vantaggi clinici, metabolici e alla eccellente riabilitazione offerta.

Fortunatamente la scelta coincide anche con i costi più contenuti.

Considero l'emodialisi giornaliera «il modo più normale di vivere e convi_ vere con la dialisi».

placida@jumpy.it

\section{BIBLIOGRAFIA}

1. Woods JD, Port FK, Stannard D, Blagg CR, Held PJ. Comparison of mortality with home hemodialysis and center hemodialysis: A national study. Kidney Int 1996; 49: 1464-70.

2. Giannattasio M. Emodialisi o peritoneodialisi come terapia di prima scelta dell'uremia cronica? Giorn Ital Nefrol 2000; 2: 161-9.

3. Pinciaroli AR. Results of daily hemodialysis in Catanzaro: 12year experience with 22 Patients treated for more than one year. Home Hemodial Int 1998; 2: 12-7.

4. Pinciaroli AR. Hormonal changes in daily hemodialysis. Seminars in Dialysis 1999; 12: 455-61.

5. Ruggieri G, Brunori G, De Vecchi A, et al. Il costo inapparente privato e sociale indotto dalla dialisi cronica. Giorn Ital Nefrol 1996; 13 (supp1): S1-36.

6. Schena FP, Biasioli S, Cavagnino A, et al. Rapporto della commissione dialisi (SIN) sui costi della terapia dialitica. Giorn Ital Nefrol 1997; 14: 321-38.

7. Morduchowicz G, Boner G. Hospitalizations in dialysis end-stage renal failure patients. Nephron 1996; 73: 413-6.

8. Catizone L, Zucchelli P. Scelta del trattamento dialitico e contenimento dei costi: ruolo dell'early referral e della dialisi peritoneale. Giorn Ital Nefrol 2000; 6: 613-23. 